TITLE:

\title{
Simple Fabrication of Silicon Nanowires by Zinc-Thermal Reduction of Silicon Tetrachloride at $773 \mathrm{~K}$
}

\section{$\operatorname{AUTHOR}(S)$ :}

Nishimura, Yusaku; Nohira, Toshiyuki; Mizutani, Yousuke; Hagiwara, Rika

\section{CITATION:}

Nishimura, Yusaku ... [et al]. Simple Fabrication of Silicon Nanowires by Zinc-Thermal Reduction of Silicon Tetrachloride at 773 K. Electrochemical and Solid-State Letters 2011, 14(11): K63.

\section{ISSUE DATE:}

2011

URL:

http://hdl.handle.net/2433/258957

\section{RIGHT:}

This is the Accepted Manuscript version of an article accepted for publication in Electrochemical and Solid-State Letters. The Electrochemical Society and IOP Publishing Ltd are not responsible for any errors or omissions in this version of the manuscript or any version derived from it. The Version of Record is available online at 
Simple fabrication of silicon nanowires by zinc-thermal reduction of silicon tetrachloride at $773 \mathrm{~K}$

Yusaku Nishimura ${ }^{\dagger}$, Toshiyuki Nohira*, Yohsuke Mizutani, and Rika Hagiwara

Graduate School of Energy Science, Kyoto University, Yoshida-hommachi, Sakyo-ku, Kyoto 606-8501, Japan

*Corresponding author: nohira@energy.kyoto-u.ac.jp

†Present address: Materials Analysis \& Evaluation Division, Toyota Central R\&D Labs, Inc., 41-1 Yokomichi, Nagakute, Aichi 480-1192, Japan.

Silicon nanowires (SiNWs) have been a target of intensive research because of their unique properties ${ }^{1-4}$ and prospective applications $^{4-6}$. New SiNW production processes that are more suitable for industrial and/or laboratory scales are strongly needed, because the conventional processes require complex apparatuses and/or procedures. Here, we report a simple SiNW production process based on zinc-thermal reduction of $\mathrm{SiCl}_{4}$ in a sealed Pyrex tube at $773 \mathrm{~K}$. Without using a catalyst, SiNWs with a diameter of about $300 \mathrm{~nm}$ were produced. The SiNWs consisted of mainly an amorphous phase, but also included a minor microcrystalline component. The introduction of Au nanoparticles to the reaction tube wall facilitated crystallization and resulted in the growth of thinner SiNWs. The typical 


\section{diameter of these SiNWs was 10-20 nm. The simple apparatus and low operating temperature of this new process is advantageous in producing SiNWs on both industrial and laboratory scales.}

Silicon nanowires are notable for their potential applications, such as SiNW-array solar cells $^{4}$, thermoelectric devices ${ }^{5}$, and negative electrodes for Li-ion batteries ${ }^{6}$. The leading processes of bottom-up fabrication (e.g., the vapor-liquid-solid (VLS) process ${ }^{7}$ and the solution-liquid-solid (SLS) process ${ }^{1}$ ) utilize droplets of metal catalysts such as Au and $\mathrm{Ag}$ for SiNW growth. The Si sources in these processes are laser ablation of bulk $\mathrm{Si}^{7}$, decomposition of polysilane ${ }^{1}$, or reduction of $\mathrm{SiCl}_{4}$ by $\mathrm{Na}^{8}$ or $\mathrm{H}_{2}{ }^{9}$. On the other hand, chemical etching ${ }^{10}$ is a leading top-down fabrication process, where $\mathrm{Si}$ wafers are etched to form nanowires with assistance from metal catalysts. Although these conventional processes are relatively successful, new production processes that require less complex apparatuses and can avoid the risk of contamination by catalysts or solvents are highly desirable.

The authors believed that zinc-thermal reduction of $\mathrm{SiCl}_{4}$ represented a promising $\mathrm{SiNW}$ production process. The zinc-thermal reduction of $\mathrm{SiCl}_{4}$ was first reported by Lyon et al. in 1949 for the production of high-purity $\mathrm{Si}^{11}$. Recently, the process has been intensively researched for the mass production of solar-grade $\mathrm{Si}^{12,13}$. It is well known that the zinc-thermal reduction of $\mathrm{SiCl}_{4}$ at $1073-1273 \mathrm{~K}$ produces $\mathrm{Si}$ fibers of micrometer-scale diameter ${ }^{14}$. The authors expected that this diameter could be reduced to a nanometer scale by lowering the reaction temperature to around $773 \mathrm{~K}$. This lowering of the temperature significantly enlarged the range of available materials for both apparatus and substrate. For example, steel and Pyrex can be used at $773 \mathrm{~K}$, but not above $1073 \mathrm{~K}$. Moreover, the low-temperature process consumes less energy. More 
recently, Uesawa et al. reported that crystalline SiNWs can be produced by zinc-thermal reduction of $\mathrm{SiCl}_{4}$ at $1193-1283 \mathrm{~K}^{15}$. However, this higher process temperature restricts the available materials and consumes more energy.

In this study, the fabrication of SiNWs was conducted by a very simple process. $\mathrm{SiCl}_{4}$ and Zn were sealed in a Pyrex tube and heated to 773 K (Figs. 1a and 1b). Further efforts were devoted to reducing the diameter of SiNWs by introducing $\mathrm{Au}$ nanoparticles (AuNPs) similar to those used in the VLS and the SLS processes, as described later (Fig. 1c).

As can be seen in Fig. 1b, a dark-brown powder was produced on the tube walls. According to the literature ${ }^{16,17}$, nanostructured $\mathrm{Si}$, such as nanowires and nanoparticles, has a dark-brown color. Figures $2 \mathrm{a}$ and $2 \mathrm{~b}$ show SEM images of the dark-brown substance captured at different magnifications. The lower-magnification image (Fig. 2a) revealed that the substance consisted of uniform nanowires. At a higher magnification (Fig. 2b), it was found that the SiNWs had an average diameter of approximately 300 $\mathrm{nm}$. In addition, the surface of the SiNWs appeared to be rough, suggesting that the formation mechanism was different from that of the VLS method. Figure 2c shows an EDX spectrum of the SiNWs. Impurities such as $\mathrm{Zn}$ and $\mathrm{Cl}$ were not detected in the SiNWs. Their microscopic structure and crystallinity were further investigated by TEM and SAED, as shown in Fig. 3a. The SiNW was comprised of two parts with different appearances: dark domains 5-20 $\mathrm{nm}$ in size and larger gray regions. The ED pattern indicated that the dark domains corresponded to crystalline $\mathrm{Si}$, and the gray regions were amorphous Si. Therefore, the produced SiNWs were composed mainly of amorphous Si and partly of nano-crystalline Si. Micro-Raman spectroscopy was performed to evaluate the crystallinity of the SiNWs. As shown in Fig. 3b, a major broad peak centered at $480 \mathrm{~cm}^{-1}$ was observed, and a small sharp peak was superimposed at $520 \mathrm{~cm}^{-1}$. There were also two small broad peaks around $300 \mathrm{~cm}^{-1}$ and 
$400 \mathrm{~cm}^{-1}$. According to the literature ${ }^{18-20}$, crystalline Si has a sharp peak assigned to optical phonons centered at $520 \mathrm{~cm}^{-1}$. On the other hand, amorphous Si has broad peaks: one assigned to the transverse-optical phonon near $480 \mathrm{~cm}^{-1}$, another assigned to the longitudinal-optical phonon around $400 \mathrm{~cm}^{-1}$, and the other assigned to the longitudinal-acoustic phonon around $300 \mathrm{~cm}^{-1}$. Their peak locations and broadness are due to the lack of long-range order and the relaxation of momentum conservation. Furthermore, for crystalline Si nanostructures, phonon confinement effects cause not only a red-shift of the peak, but also asymmetric peak broadening ${ }^{21,22}$. Therefore, Raman spectroscopy confirmed that the SiNWs consisted mainly of amorphous Si and partly of nano-crystalline Si. Amorphous SiNWs are considered to be favorable for the application as an anode material for Li-ion batteries ${ }^{23}$.

Zinc-thermal reduction has been widely investigated for the mass production of solargrade Si and can be expressed as ${ }^{11-14}$

$$
\mathrm{SiCl}_{4}(\mathrm{~g})+2 \mathrm{Zn}(\mathrm{g}) \rightarrow \mathrm{Si}(\mathrm{s})+2 \mathrm{ZnCl}_{2}(\mathrm{~g})
$$

Since the typical operation temperature is $1073-1273 \mathrm{~K}$ during solar-grade $\mathrm{Si}$ production, few studies of zinc-thermal reduction at $773 \mathrm{~K}$ have been performed, and there has been no report on the formation mechanism of SiNWs at 773 K. Figure 4 shows our explanation of the SiNW formation mechanism at $773 \mathrm{~K}$. As shown in Fig. 4a, the gasification of $\mathrm{SiCl}_{4}$ (b.p. $331 \mathrm{~K}$ ) occurs, followed by the melting of $\mathrm{Zn}$ (m.p. $693 \mathrm{~K}$ ). Then, the reaction of $\mathrm{SiCl}_{4}$ and $\mathrm{Zn}$ vapor begins in the vicinity of liquid $\mathrm{Zn}$. Since the vapor pressure of $\mathrm{Zn}$ is relatively low at $773 \mathrm{~K}$, approximately $1.5 \times 10^{2} \mathrm{~Pa}$, the reaction front gradually progresses along the tube wall from the bottom to the top. The formation of SiNWs at the tube wall is schematically shown in Fig. 4b. As is often observed during CVD, the initial deposition of Si particles occurs preferentially at a solid substrate, which was the tube wall in the present case. Then, the produced Si 
particles serve as a catalyst for subsequent reaction. Considering the low vapor pressure of $\mathrm{Zn}$, the reaction sites were in a state of $\mathrm{Zn}$ depletion, causing the Si to grow toward the bulk in the shape of nanowires.

As described above, SiNWs were formed by zinc-thermal reduction of $\mathrm{SiCl}_{4}$ at $773 \mathrm{~K}$ without a catalyst. However, the diameter of the SiNW was relatively large (about 300 $\mathrm{nm})$. To reduce the diameter of SiNWs in the same manner as in the VLS process, AuNPs were introduced to the tube sidewall as a catalyst. After heating to $773 \mathrm{~K}$, a large amount of brown substance was deposited on the tube sidewall (Fig. 1c). Figures $5 \mathrm{a}$ and $5 \mathrm{~b}$ show SEM images of the brown deposits, in which SiNWs 10-100 nm in diameter, but typically $20-30 \mathrm{~nm}$ in diameter, were observed. According to EDX analysis (Supplementary Fig. S1), a distinct peak of the Au M $\alpha$ line was observed only at the tip of the SiNWs. This suggests that the AuNPs did act as catalysts, in the same manner as in the VLS process.

The microscopic structure and crystallinity of SiNWs produced in the presence of AuNPs were also investigated. Figure 5c shows a TEM image and an SAED pattern obtained from these samples. The diameter of the SiNWs was approximately $10 \mathrm{~nm}$, and the crystallinity was very high. The formation of crystalline SiNWs was further supported by micro-Raman spectroscopy, as shown in Fig. $5 \mathrm{~d}$, because a prominent peak centered at $520 \mathrm{~cm}^{-1}$, which was assigned to crystalline Si, was clearly observed.

Although the addition of AuNPs is not practical for large-scale production because of their high cost, other inexpensive catalysts such as $\mathrm{Al}$, In, and $\mathrm{Sn}^{24}$ may also be effective for the present $\mathrm{Zn}-\mathrm{SiCl}_{4}$ system.

\section{Methods}


Liquid $\mathrm{SiCl}_{4}$ (purity: 99.9\%, Wako Pure Chemical Industries, Ltd.) and Zn grains (purity: 99.9\%, diameter: 75-150 $\mu \mathrm{m}$, Wako Pure Chemical Industries, Ltd.) were introduced to a Pyrex tube (1 cm in diameter and $10 \mathrm{~cm}$ in length) in a glove box or glove bag filled with high-purity Ar gas. Typical amounts of $\mathrm{SiCl}_{4}$ and $\mathrm{Zn}$ were $0.2 \mathrm{~mL}$ $\left(1.7 \times 10^{-3} \mathrm{~mol}\right)$ and $0.4 \mathrm{~g}\left(6.1 \times 10^{-3} \mathrm{~mol}\right)$, respectively. In some experiments, AuNPs (diameter: 20-30 $\mu \mathrm{m}$, Sumitomo Electric Industries, Ltd.) were placed on the inner wall of the glass tube beforehand. Then, the glass tube was sealed with a gas burner and placed in an electric furnace. The glass tube was heated to $773 \mathrm{~K}$ for 48 hours. After cooling, the tube was cut, and a powdery substance was scraped from the inner wall and rinsed with distilled water and ethanol. The dried powder was then characterized and analyzed by the following methods.

First, the morphology of the substance was observed with a field-emission-type scanning electron microscope (FE-SEM, JEOL Ltd., JSM-6500F). Energy-dispersive Xray spectroscopy (EDX, JEOL Ltd., JSM-6500FE) was performed to investigate the composition of the observed substance. The microscopic structure and the crystallinity of the produced SiNWs were studied by transmission electron microscopy (TEM, Hitachi, H-9000UHR) and selected area electron diffraction (SAED), respectively. Local analysis was carried out by micro-Raman spectroscopy (Horiba Jobin Yvon Inc., Labram010) using a He-Ne laser $(632.8 \mathrm{~nm})$. It should be noted that the micro-Raman spectroscopic analysis of the reaction product was conducted directly through the tube wall before cutting and exposing the sample to air. Laser heating during the measurement could cause not only the crystallization of an initially amorphous substance, but also an anharmonic thermal effect on the peak shape. Therefore, the temperature at the measurement point was estimated from the intensity ratio of Stokes to anti-Stokes scattering and the laser output was carefully adjusted so as not to heat up the measurement point. 


\section{References}

1 Heitsch, A. T., Fanfair, D. D., Tuan, H.-Y. \& Korgel, B. A. Solution-liquidsolid (SLS) growth of silicon nanowires. Journal of the American Chemical Society 130, 5436-5437 (2008).

2 Lu, W. \& Lieber, C. M. Semiconductor nanowires. Journal of Physics D: Applied Physics 39, R387-R406 (2006).

3 Ball, P. Silicon in optoelectronics. Let there be light. Nature 409, 974-976 (2001).

4 Tsakalakos, L. et al. Silicon nanowire solar cells. Applied Physics Letters 91, 233117/233111-233117/233113 (2007).

5 Shi, L., Yao, D., Zhang, G. \& Li, B. Size dependent thermoelectric properties of silicon nanowires. Applied Physics Letters 95, 063102/063101-063102/063103 (2009).

6 Chan, C. K. et al. High-performance lithium battery anodes using silicon nanowires. Nature Nanotechnology 3, 31-35 (2008).

7 Morales, A. M. \& Lieber, C. M. A laser ablation method for the synthesis of crystalline semiconductor nanowires. Science (Washington, D. C.) 279, 208-211 (1998).

8 Wang, C., Jiang, Y., Li, G. \& Zhang, Z. A wurtz-like reaction to silicon nanowires. Materials Letters 62, 2497-2499 (2008).

9 Hochbaum, A. I., Fan, R., He, R. \& Yang, P. Controlled growth of Si nanowire arrays for device integration. Nano Lett. 5, 457-460 (2005).

10 Huang, Z. et al. Extended arrays of vertically aligned sub-10 nm diameter [100] Si nanowires by metal-assisted chemical etching. Nano letters 8 , 3046-3051 (2008).

11 Lyon, D. W., Olson, C. M. \& Lewis, E. D. Preparation of hyper-pure silicon. Journal of the Electrochemical Society 96, 359-363 (1949).

12 Honda, S., Yasueda, M., Hayashida, S., Yamaguchi, M. \& Tanaka, T. Production process for high purity polycrystal silicon and production apparatus for the same. US Patent 2007123011 (2007).

13 Yasuda, K. \& Okabe, T. H. Production processes of solar grade silicon based on metallothermic reduction. Nippon Kinzoku Gakkaishi 74, 1-9 (2010).

14 Johnson, E. R. \& Amick, J. A. Formation of single-crystal silicon fibers. Journal of Applied Physics 25, 1204-1205 (1954).

15 Uesawa, N., Inasawa, S., Tsuji, Y. \& Yamaguchi, Y. Gas-Phase Synthesis of Rough Silicon Nanowires via the Zinc Reduction of Silicon Tetrachloride. Journal of Physical Chemistry C 114, 4291-4296 (2010).

16 Nohira, T., Yasuda, K. \& Ito, Y. Pinpoint and bulk electrochemical reduction of insulating silicon dioxide to silicon. Nature Materials 2, 397401 (2003).

17 Scriba, M. R., Arendse, C., Haerting, M. \& Britton, D. T. Hot-wire synthesis of Si nanoparticles. Thin Solid Films 516, 844-846 (2008). 
Zwick, A. \& Carles, R. Multiple-order Raman scattering in crystalline and amorphous silicon. Physical Review B: Condensed Matter and Materials Physics 48, 6024-6032 (1993).

19 Temple, P. A. \& Hathaway, C. E. Multiphonon Raman spectrum of silicon. Physical Review B: Solid State 7, 3685-3697 (1973).

20 Brodsky, M. H., Cardona, M. \& Cuomo, J. J. Infrared and Raman spectra of the silicon-hydrogen bonds in amorphous silicon prepared by glow discharge and sputtering. Physical Review B: Solid State 16, 3556-3571 (1977).

21 Richter, H., Wang, Z. P. \& Ley, L. The one phonon Raman spectrum in microcrystalline silicon. Solid State Communications 39, 625-629 (1981).

22 Campbell, I. H. \& Fauchet, P. M. The effects of microcrystal size and shape on the one phonon Raman spectra of crystalline semiconductors. Solid State Communications 58, 739-741 (1986).

23 Hatchard, T. D. \& Dahn, J. R. In Situ XRD and Electrochemical Study of the Reaction of Lithium with Amorphous Silicon. Journal of the Electrochemical Society 151, A838-A842 (2004).

$24 \mathrm{Yu}, \mathrm{L}$. et al. Plasma-enhanced low temperature growth of silicon nanowires and hierarchical structures by using tin and indium catalysts. Nanotechnology 20, 225604 (2009).

Figure Legends

Figure 1. Photographs of (a) the original Pyrex tube containing $\mathrm{SiCl}_{4}$ and $\mathrm{Zn}$ grains, (b) the same tube after heating at $773 \mathrm{~K}$ for 48 hours. In (c), Au nanoparticles were placed on the inner wall of the glass tube beforehand.

Figure 2. (a), (b) SEM images of SiNWs produced by zinc-thermal reduction of $\mathrm{SiCl}_{4}$ at $773 \mathrm{~K}$ taken at different magnifications. (c) An EDX spectrum of SiNWs.

Figure 3. (a) A TEM image (inset: an SAED pattern) and (b) a Raman spectrum of $\mathrm{SiNWs}$ produced by zinc-thermal reduction of $\mathrm{SiCl}_{4}$ at $773 \mathrm{~K}$.

Figure 4. A formation mechanism of SiNWs by zinc-thermal reduction of $\mathrm{SiCl}_{4}$ in a sealed glass tube at $773 \mathrm{~K}$. (a) The phase change of materials during the 
heating process and the progress of SiNW formation at $773 \mathrm{~K}$. (b) A schematic representation of SiNW formation at the tube wall.

Figure 5. (a), (b) SEM images at different magnifications, (c) a TEM image (inset: an SAED pattern) and (d) a Raman spectrum of SiNWs produced by zinc-thermal reduction of $\mathrm{SiCl}_{4}$ in the presence of AuNPs at $773 \mathrm{~K}$.

Figure S1. An EDX spectrum measured at the tip of a SiNW that was produced by zinc-thermal reduction of $\mathrm{SiCl}_{4}$ in the presence of AuNPs at $773 \mathrm{~K}$. 
(a)

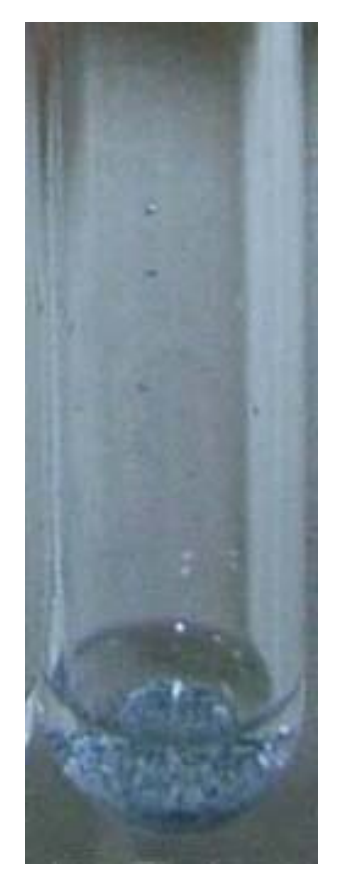

(b)

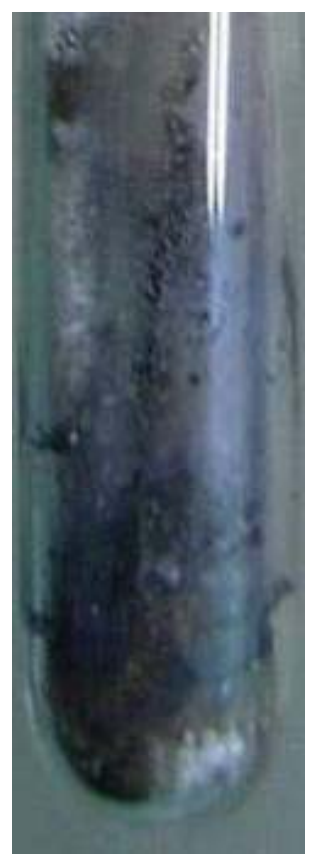

(c)

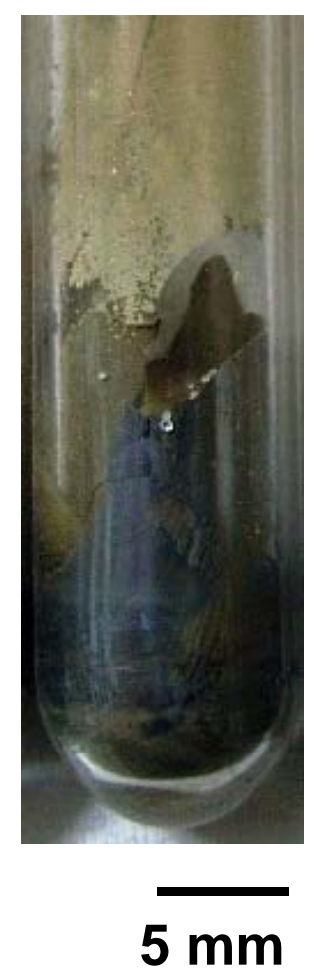

Figure 1. Photographs of (a) the original Pyrex tube containing $\mathrm{SiCl}_{4}$ and $\mathrm{Zn}$ grains, (b) the same tube after heating at $773 \mathrm{~K}$ for 48 hours. In (c), Au nano-particles were placed on the inner wall of the glass tube beforehand. 
(a)

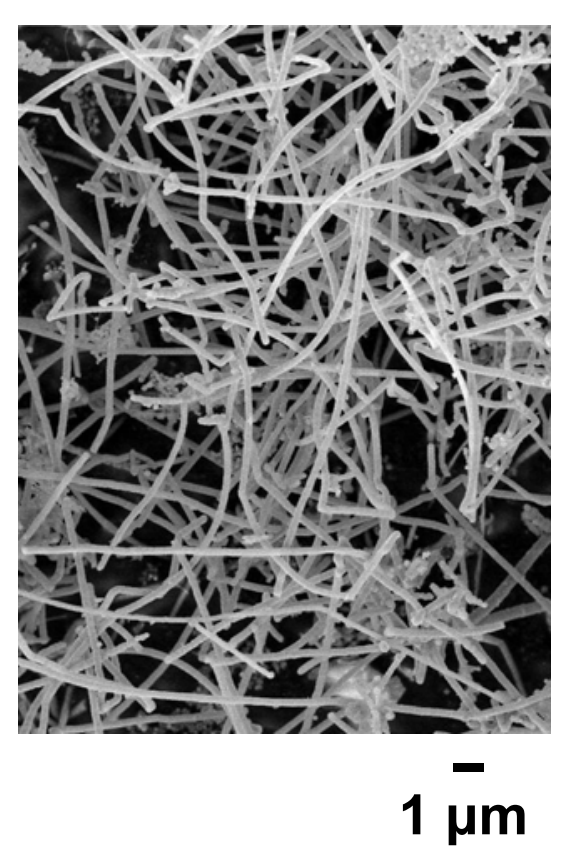

(b)

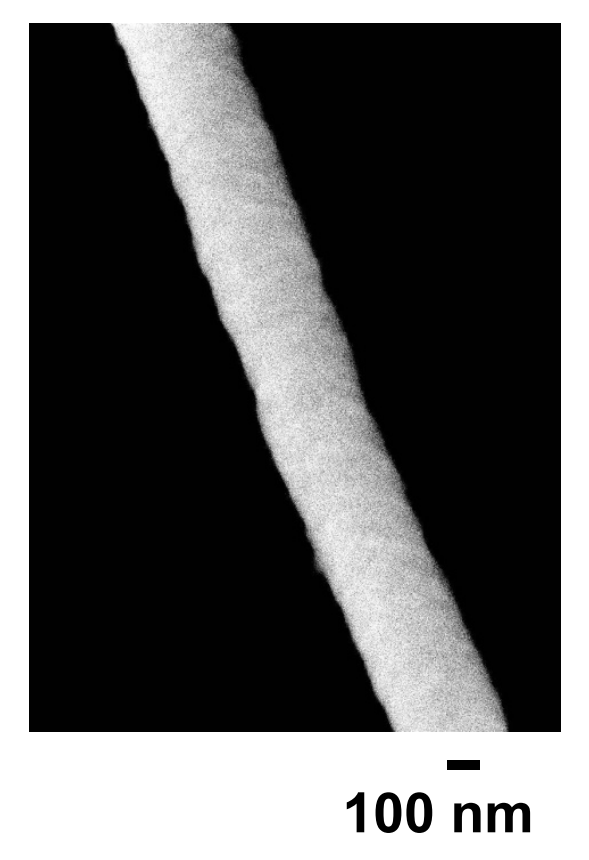

(c)

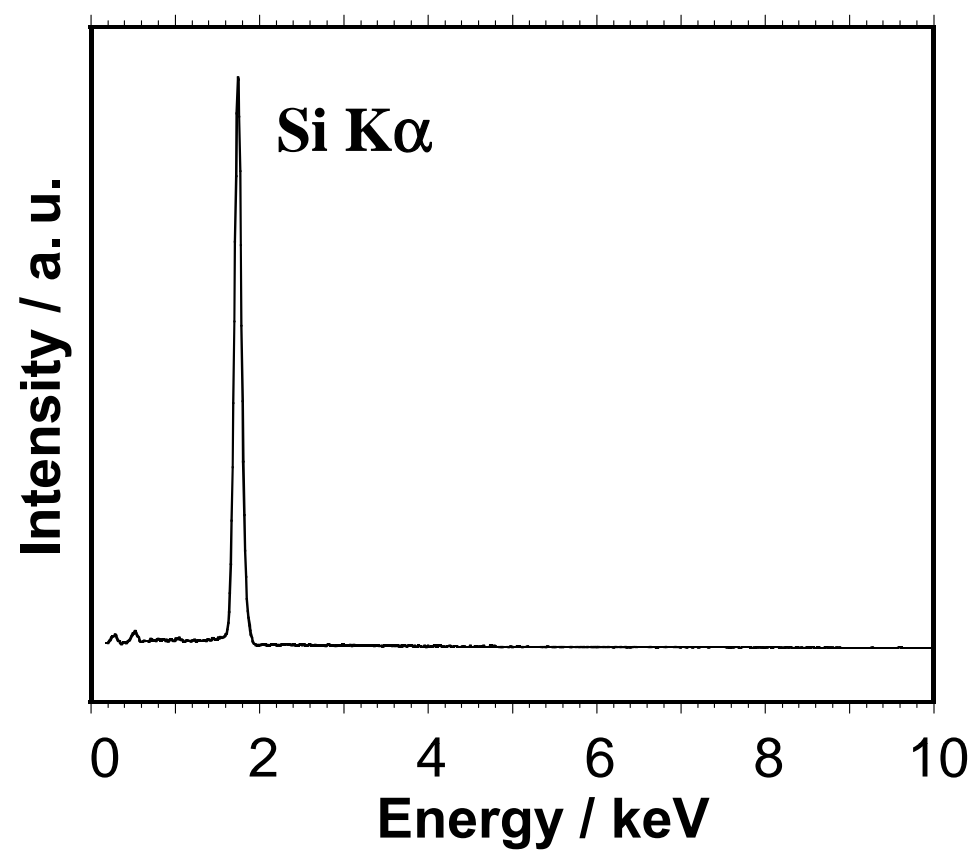

Figure 2. (a), (b) SEM images of SiNWs produced by zinc-thermal reduction of $\mathrm{SiCl}_{4}$ at $773 \mathrm{~K}$ taken at different magnifications. (c) An EDX spectrum of SiNWs. 
(a)

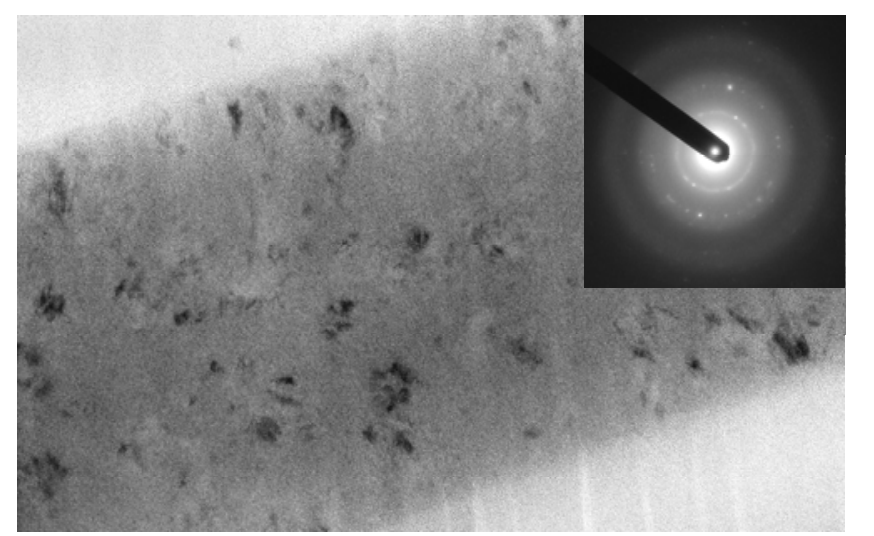

(b)

$40 \mathrm{~nm}$

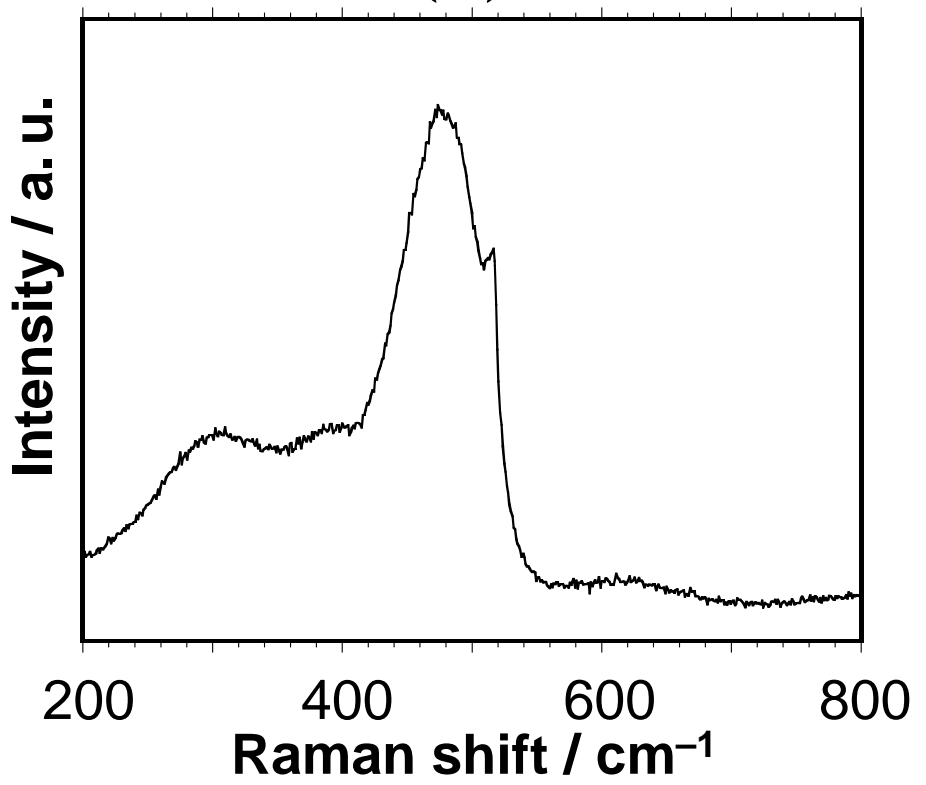

Figure 3. (a) A TEM image (inset: an SAED pattern) and (b) a Raman spectrum of SiNWs produced by zinc-thermal reduction of $\mathrm{SiCl}_{4}$ at $773 \mathrm{~K}$. 
(a)

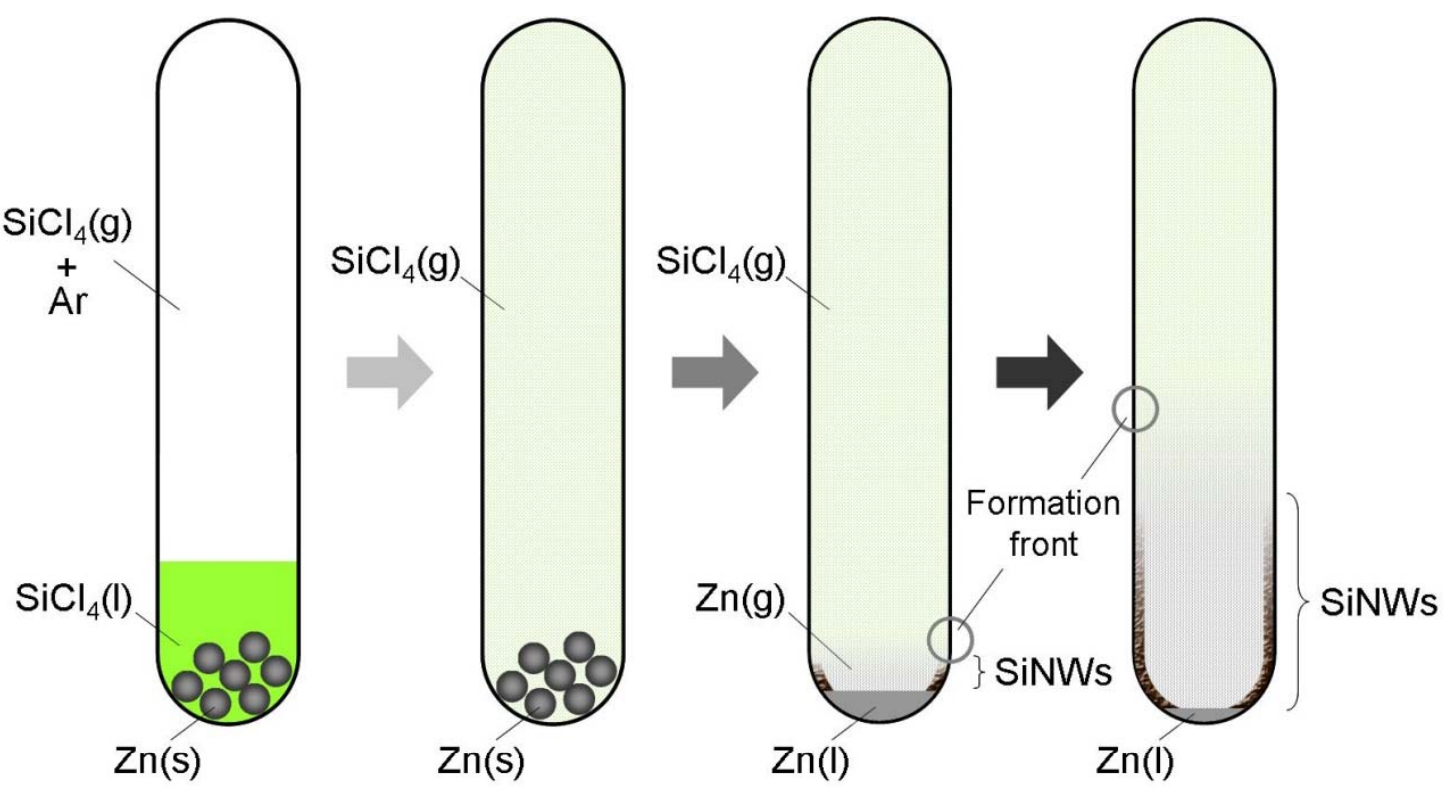

$$
T=298 \mathrm{~K} T=673 \mathrm{~K} \quad T=773 \mathrm{~K} \quad T=773 \mathrm{~K}
$$

(b)
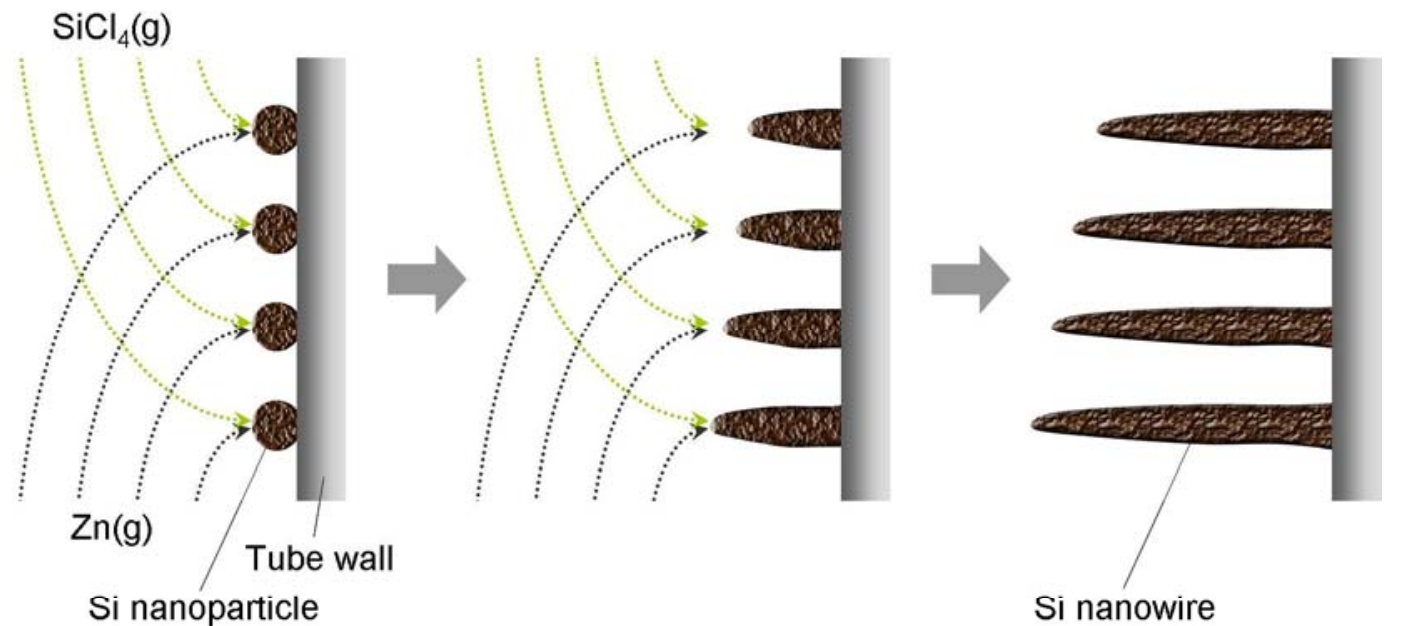

Figure 4. A formation mechanism of SiNWs by zinc-thermal reduction of $\mathrm{SiCl}_{4}$ in a sealed glass tube at $773 \mathrm{~K}$. (a) The phase change of materials during the heating process and the progress of SiNW formation at $773 \mathrm{~K}$. (b) A schematic representation of SiNW formation at the tube wall. 
(a)

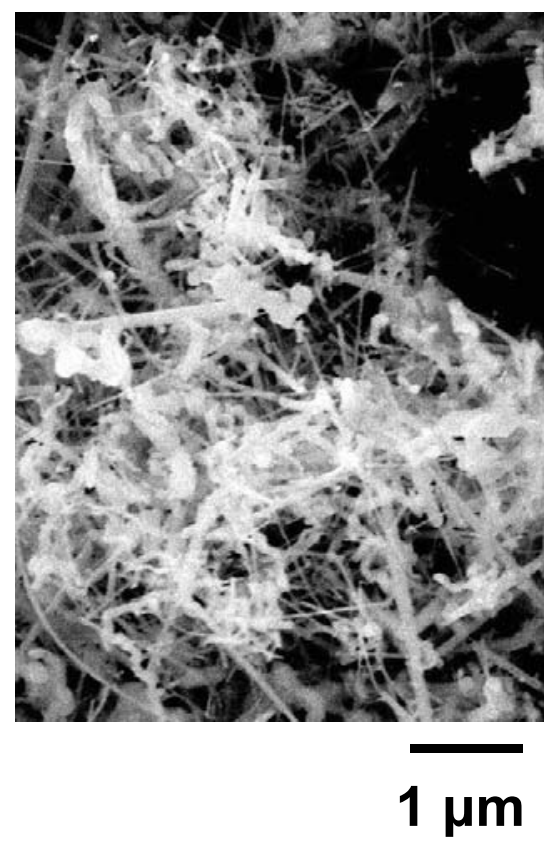

(b)

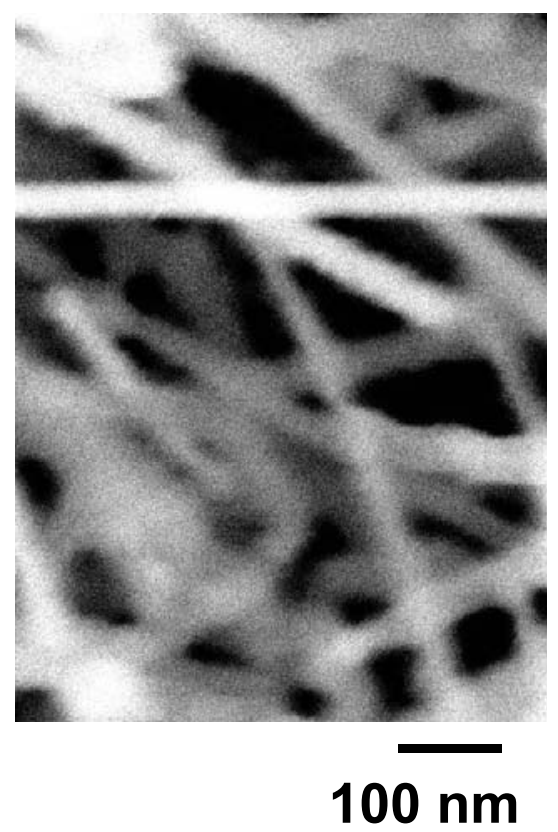

(c)

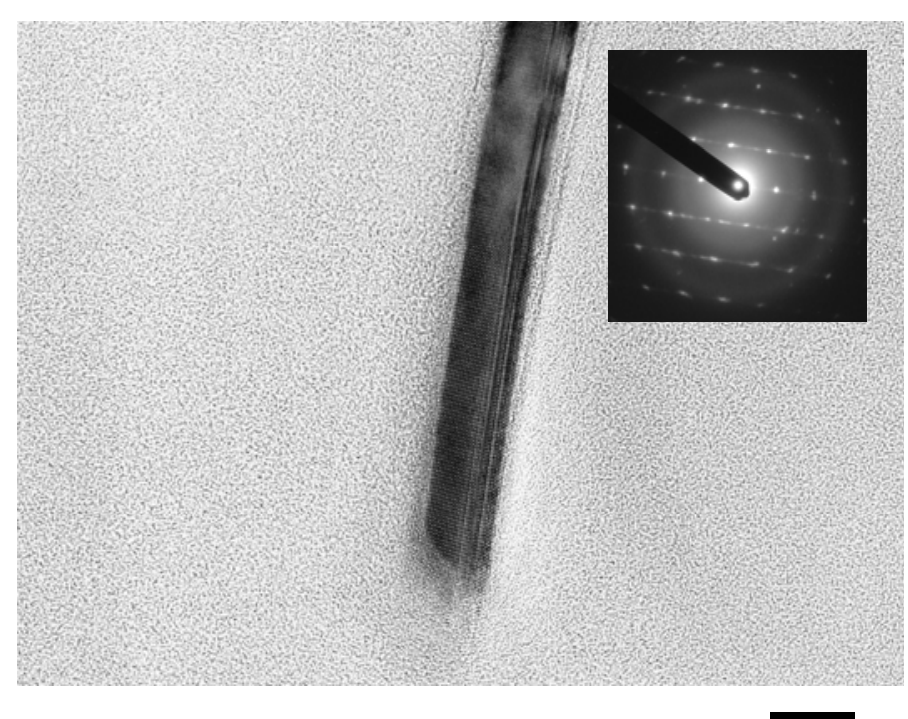

$10 \mathrm{~nm}$

(d)

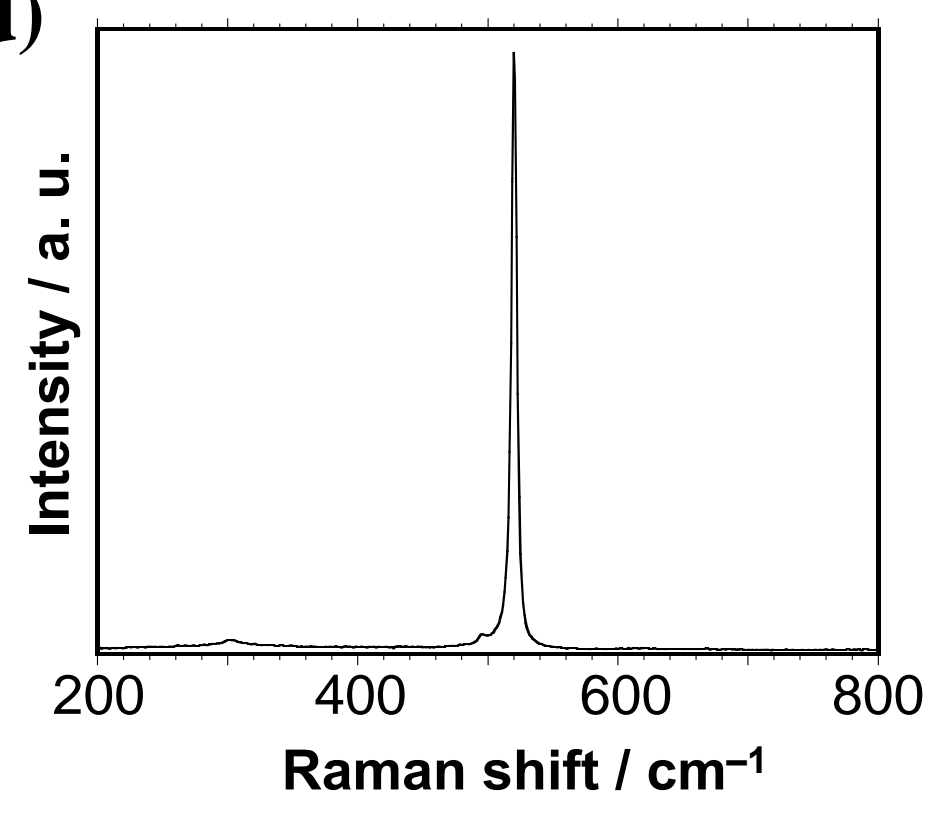

Figure 5. (a), (b) SEM images at different magnifications, (c) a TEM image (inset: an SAED pattern) and (d) a Raman spectrum of SiNWs produced by zinc-thermal reduction of $\mathrm{SiCl}_{4}$ in the presence of AuNPs at $773 \mathrm{~K}$. 


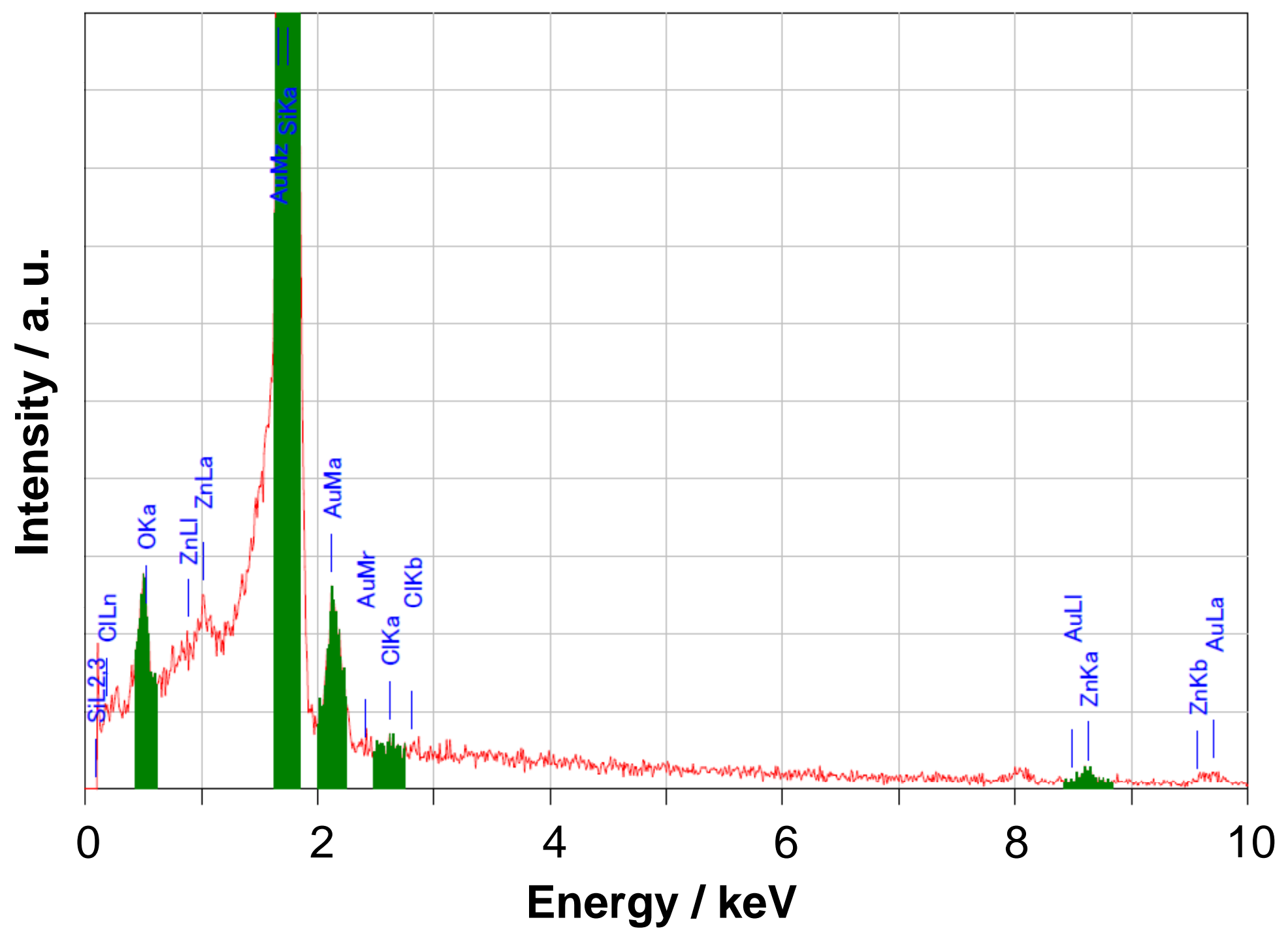

Figure S1. An EDX spectrum measured at the tip of a SiNW that was produced by zinc-thermal reduction of $\mathrm{SiCl}_{4}$ in the presence of AuNPs at $773 \mathrm{~K}$. 